\title{
Análisis de opciones reales para la valoración financiera de proyectos de energía geotérmica en Colombia *
}

\author{
Real Options Analysis for Financial Valuation of Geothermal Energy Projects in \\ Colombia
}

\author{
Yessenia Martínez-Ruiz \\ Doctorando en Ingeniería, Universidad del Valle, \\ Cali - Colombia, yessenia.martinez@correounivalle.edu.co \\ Diego Fernando Manotas-Duque \\ Doctor en Ingeniería, Universidad del Valle, \\ Cali -Colombia,diego.manotas@correounivalle.edu.co \\ Howard Ramírez-Malule \\ Doctor en Ingeniería Química, Universidad del Valle, \\ Cali - Colombia, howard.ramirez@correounivalle.edu.co
}

\begin{abstract}
Cómo citar / How to cite
Martínez-Ruiz, Y., Manotas-Duque, D. F., Ramírez-Malule, H. (2021). Análisis de opciones reales para la valoración financiera de proyectos de energía geotérmica en Colombia. Revista CEA, v. 7, n. 15, e1944. https://doi.org/10.22430/24223182.1944
\end{abstract}

Recibido: 23 de marzo de 2021

Aceptado: 12 de julio de 2021

\section{Resumen}

Los proyectos de inversión en energía frecuentemente son evaluados mediante la aplicación de técnicas clásicas que asumen un panorama estático en los flujos de caja, resultando insuficientes al no considerar los riesgos asociados a las variables de entrada ni la flexibilidad de los inversionistas para la toma de decisiones. El objetivo principal de esta investigación fue evaluar la viabilidad financiera de proyectos de energía geotérmica en Colombia, considerando la incertidumbre y el riesgo característico de este tipo de proyectos. Primero, se describió el caso de estudio analizado, las variables de modelación más comunes en proyectos de esta índole y las distribuciones probabilísticas asociadas. Posteriormente se utilizó la simulación de Monte Carlo para conocer la viabilidad financiera del proyecto mediante la aplicación de criterios clásicos de la literatura. Estos hallazgos

\footnotetext{
*Este artículo se deriva del proyecto titulado «Tópicos de Investigación en Valoración de Inversiones en Energía Renovable»
} y ha sido financiado con recursos de la Universidad del Valle. 
fueron comparados con los obtenidos al aplicar el enfoque de opciones reales al caso de estudio, el cual fue evaluado a partir de una opción real compuesta durante las fases de inversión. Los resultados obtenidos evidenciaron que mediante el uso de los métodos tradicionales el proyecto fue clasificado como no factible, pero, al considerar la flexibilidad de los inversionistas, este resultó atractivo. Por tanto, al momento de evaluar la viabilidad financiera de un proyecto de inversión se deben considerar factores de riesgo e incertidumbre, los cuales agregan valor al análisis de factibilidad y presentan una visión más cercana a la realidad de los proyectos.

Palabras clave: opciones reales, riesgo de capital, probabilidad financiera, fuentes de energía geotérmica.

Clasificación JEL: Q2, Q4.

\title{
Highlights
}

- El análisis financiero tradicional subestima el valor de los proyectos geotérmicos.

- La generación geotérmica en Colombia debe considerar la incertidumbre y el riesgo financiero asociado a este tipo de proyectos.

- El análisis de opciones reales facilita la evaluación del riesgo financiero en proyectos geotérmicos.

\begin{abstract}
Energy investment projects are usually valuated applying classical techniques that assume static cash flows. However, such techniques are insufficient because they do not consider the risks associated with the input variables or the decision-making flexibility of investors. This study evaluates the financial viability of geothermal energy projects in Colombia while considering uncertainty and the characteristic risk of this kind of projects. It describes the study case under analysis, the most common modeling variables used in projects of this type, and the associated probabilistic distributions. A Monte Carlo simulation was implemented to establish the financial viability of a project by applying classical criteria found in the literature. The findings were compared to those obtained when the real options approach was applied to the study case, which was evaluated based on a compound real option during the investment phases. The results show that the project is not feasible when traditional valuation methods are used, but it is attractive when investor flexibility is taken into account. Therefore, risk factors and uncertainty should be considered in the evaluation of the financial viability of investment projects because they add value to the feasibility analysis and offer a more realistic vision of such projects.
\end{abstract}

Keywords: Real options, capital risk, financial probability, geothermal energy sources.

JEL classification: Q2, Q4.

\section{Highlights}

- Traditional financial analysis underestimates the value of geothermal projects.

- Geothermal energy generation in Colombia should consider the uncertainty and financial risk associated with this kind of projects.

- Real options analysis facilitates the evaluation of financial risk in geothermal projects. 


\section{INTRODUCCIÓN}

El interés por el uso de fuentes de energía limpia ha ido en constante aumento en algunos países (Gavrikova et al., 2019), producto de las preocupaciones ambientales y la dependencia de recursos fósiles (Agaton, 2017; Daneshvar et al., 2021). Colombia no es ajena a este comportamiento. Recientemente las políticas regulatorias del país han estado orientadas a promover proyectos de inversión en fuentes no convencionales de energías renovables (FNCER) mediante la creación de incentivos económicos y tributarios (Córdoba Cárdenas et al., 2019) con el fin de diversificar la matriz energética. Estos incentivos vienen dados especialmente por cambios en el panorama regulatorio del país, como la introducción de la Ley 1715 , por medio de la cual se regula la integración de las energías renovables no convencionales al Sistema Energético Nacional (Unidad de Planeación Minero Energética [UPME], 2015), y la Resolución 030 de 2018 de la Comisión de Regulación de Energía, Gas y Combustibles (CREG, 2018), la cual permite la autogeneración energética a pequeña escala y la generación distribuida al Sistema Interconectado Nacional (SIN).

Sin embargo, es importante destacar que los proyectos en energía renovable se caracterizan por su alto riesgo e incertidumbre (Li et al., 2020; Ju et al., 2019), lo que hace que al momento de evaluar financieramente su viabilidad sea necesario considerar dichas características, las cuales afectan la evolución de los flujos de efectivo de dichos proyectos (Bilqist et al., 2018), influyendo, además, en la elección del mejor método para evaluar las inversiones en energía (Santos et al., 2014). La manera en que los inversionistas deben evaluar este tipo de inversiones es considerando los factores adicionales de riesgo e incertidumbre (Rocha et al., 2018), haciendo que la valoración de estos proyectos, mediante métodos tradicionales como el análisis de flujo de caja, el valor presente neto (VPN) y la tasa interna de retorno (TIR), entre otros, resulte insuficiente y sea necesario el uso de técnicas de evaluación más sofisticadas (Fernandes et al., 2011).

Tal como lo expresa Gonima Mena (2017), los proyectos relacionados con la energía eléctrica presentan características inherentes a esta clase de negocios, como lo son: ambiente cambiante, alto riesgo, marco regulatorio y demanda energética. Adicionalmente, los proyectos de energía renovable tienen un riesgo relevante asociado al cambio climático y la rapidez con la que cambian las tecnologías de este tipo de fuentes de energía (Bandoc et al., 2013; Kim et al., 2017). Estos aspectos no son capturados mediante los métodos tradicionales de evaluación financiera, por lo tanto, no son adecuados para la toma de decisiones con respecto a la inversión en energía renovable (Kim et al., 2017), pues la aplicabilidad de métodos clásicos para la valoración financiera disminuye en proyectos que se caracterizan por tener altos niveles de riesgo e incertidumbres (Manotas Duque y Manyoma, 2001).

El presente trabajo tuvo como principal objetivo evaluar la viabilidad financiera de proyectos de energía geotérmica en Colombia, considerando la incertidumbre y el riesgo propios de esta clase de proyectos. Los análisis fueron realizados a partir de un caso de estudio, cuyo horizonte de evaluación considera un total de 28 años (a partir del año cero): 8 años para el desarrollo de las fases de inversión y 20 años para el periodo de operación del proyecto. En primera medida se realizó la identificación de las variables de entrada más comunes e influyentes para la valoración de proyectos de esta índole, definiendo los patrones de modelación de estas para el cálculo del flujo de caja libre (FCF). Este 
trabajo utilizó la simulación Monte Carlo para analizar diferentes escenarios de valoración y la factibilidad financiera de este proyecto mediante el uso de métodos clásicos. Finalmente, la aplicación del enfoque de opciones reales fue realizada para conocer la viabilidad económica del proyecto caso de estudio, considerando la opción de abandono del proyecto durante sus fases de inversión: exploración, perforación y desarrollo mediante la aplicación de opciones reales compuestas.

Esta investigación está compuesta por 6 apartados. En el primero de ellos se realiza una introducción del tema de investigación y problemática de estudio; después se presenta una revisión de los principales métodos de valoración asociados a los proyectos de energía renovable, incluido el enfoque desarrollado en el presente artículo. Luego, la metodología empleada en la investigación a partir de la aplicación de un caso de estudio. Posteriormente se dan a conocer los resultados obtenidos. Finalmente se presenta la discusión y las principales conclusiones, y un Anexo que contiene el detalle del flujo de caja del proyecto para algunos de sus años de operación.

\section{MARCO TEÓRICO}

Santos et al. (2014) reportaron que existen diferentes técnicas para evaluar proyectos de inversión en generación energética, agrupándolos entre los métodos tradicionales y aquellos que consideran la incertidumbre como un factor que tiene influencia en la evaluación financiera de los proyectos.

La tasa interna de retorno (TIR), el valor presente neto (VPN) y el costo nivelado de energía (LCOE, por sus siglas en inglés), son clasificados como métodos tradicionales dentro de la literatura (Santos et al., 2014). Una aplicación de dos de estos métodos fue realizada por Fallah et al. (2018), quienes se enfocaron en los diferentes tipos de plantas geotérmicas desde el punto de vista técnico para conocer la viabilidad financiera de estas a partir del uso de la TIR y el LCOE. Por su parte, Lesmana et al. (2020) evaluaron la factibilidad financiera de un proyecto geotérmico en Indonesia haciendo uso del enfoque probabilístico de Monte Carlo y mediante la aplicación de los criterios de la TIR y el VPN. A pesar de ser un criterio que se ha utilizado durante muchos años para decisiones de inversión en proyectos de energía, el VPN no es considerado un método apropiado para proyectos que son altamente inciertos, pues este trae consigo un gran supuesto relacionado con flujos de caja definidos (Kim et al., 2017). Desde el punto de vista de la viabilidad financiera de cualquier proyecto, los modelos o criterios de decisión tradicionales asumen un panorama estático en la ocurrencia de las magnitudes a lo largo de la vida útil de los proyectos, resultando insuficiente al momento de percibir características relevantes para la toma de decisiones en inversiones de alto riesgo (Martínez-Ruiz et al., 2020).

De acuerdo con Brach (2003), la evaluación tradicional asume flujos rígidos en la vida de un proyecto e ignora los riesgos y la flexibilidad que tiene la gerencia para reaccionar a las diferentes situaciones/ incertidumbres que se puedan presentar. Adicionalmente, para el caso de las energías renovables, muchos estudios destacan la importancia de analizar el impacto de las políticas que promueven el uso de este tipo de energías y las regulaciones ambientales (Liu et al., 2019). 
Las opciones reales, por su parte, tienen su base conceptual en las opciones financieras al aplicarlas en el análisis de activos reales o físicos (Mun, 2002; Zhang et al., 2020). Según Damodaran (2005), una opción se define como el derecho, mas no la obligación, para comprar (opción de compra) o vender (opción de venta) una cantidad específica de un activo a un precio pactado (precio de ejercicio). Dicho activo se conoce como «activo subyacente» y se define como el activo financiero sobre el cual está valorado el instrumento financiero. De manera análoga a las opciones financieras, una compañía que cuenta con una opción real tiene el derecho - pero no la obligación - de realizar una inversión que se ve como generadora de valor (Mauboussin, 1999).

En contraste a las limitaciones de los métodos tradicionales, la aplicación de esta metodología a los proyectos de inversión considera la cuantificación del riesgo y las flexibilidades de estos, dando un valor agregado en la evaluación financiera (García Espinoza, 2016). En otras palabras, permite evaluar los diferentes escenarios de acción sobre los que puede decidir un inversionista para obtener resultados más favorables, entre los cuales se encuentra retrasar o posponer una decisión (Agaton, 2017), e incluso, abandonar el proyecto cuando este no resulta favorable (Manotas Duque y Manyoma, 2001). Estas características han convertido las opciones reales como una herramienta de gran aplicabilidad en los mercados de energía, los cuales se caracterizan por su alto riesgo e incertidumbre, especialmente en aspectos como activos de generación, evaluación de políticas e investigaciones asociadas al sector energético (Isaza Cuervo y Botero Botero, 2014).

Una aplicación interesante la realizaron Knaut et al. (2013) quienes utilizaron el enfoque de opciones reales para conocer el impacto de la incertidumbre asociada a la variable temperatura, sobre la factibilidad de un proyecto de inversión en energía geotérmica. Otras investigaciones, como la de Kim et al. (2017), hicieron uso de las opciones reales para valorar un proyecto de energía renovable en Indonesia. En este estudio, los autores aplican el modelo de opción compuesta durante las fases de planeación, diseño y construcción del proyecto, encontrando que el proyecto toma valor y reduce su riesgo, al tener la opción de abandonarlo al final de cada fase.

Las opciones reales también son utilizadas en el campo energético para la valoración de activos de energía y para la selección de portafolios de proyectos, esto se ve reflejado en Abadie (2009) y en Fernandes et al. (2011). Por otro lado, el uso de opciones reales también se ha aplicado para la valoración de proyectos en otras energías renovables. Tal es el caso de Li et al. (2018), quienes a partir de este enfoque evaluaron un proyecto de generación de energía fotovoltaica en China, considerando la incertidumbre en factores como costos de generación, precios del carbón y subsidios gubernamentales.

\section{METODOLOGÍA}

\section{Caso de estudio y variables de entrada}

Colombia cuenta con un alto potencial geotérmico producto de su ubicación, que le permite contar con las cordilleras Occidental y Central, de alta actividad volcánica (Coviello, 2000), como fuente geotérmica fundamental para el desarrollo de proyectos de generación a partir de este recurso. La metodología desarrollada en este trabajo presenta un estudio analítico basado en simulación 
computacional con Monte Carlo (in silico) para conocer la factibilidad financiera de un caso de estudio, cuya ubicación fue establecida en el departamento de Caldas, Colombia. Primero, se definieron las variables de entradas técnicas y financieras y las distribuciones de probabilidad correspondientes a cada una de ellas. Segundo, se desarrolló el modelo de factibilidad financiera para el cálculo del flujo de caja libre del proyecto. Posteriormente, la aplicación de métodos clásicos de la literatura como VPN y TIR fue realizada con el fin de conocer los criterios de decisión asociados a estos dos enfoques. Por último, se evaluó una opción real de abandono para valorar la flexibilidad del inversionista durante el periodo de inversión.

La vida útil de este proyecto considera un horizonte de 27 años. En los primeros 8, contados a partir del año 0, se desarrollan las fases de inversión: exploración (3 años), perforación ( 2 años) y desarrollo (3 años), y, por último, un periodo de operación de 20 años. Las variables de entrada fueron definidas de acuerdo con una distribución probabilística normal, uniforme y triangular, tal como se puede apreciar en las Tabla 1 y Tabla 2. Dentro de estas variables están consideradas características técnicas de los proyectos geotérmicos como lo son la temperatura del pozo $\left(T_{i}\right)$, el caudal del fluido $(\mathrm{Q})$ y el factor de planta $(f p)$ de estas tecnologías, las cuales determinan la capacidad instalada del proyecto. El precio de los contratos $\left(P_{\text {contratos }}\right)$, precio spot $\left(P_{\text {spot }}\right)$ y contrato bilateral $\left(E_{\text {contratos }}\right)$ son variables de entrada definidas acorde al entorno energético de Colombia, y representan el valor al cual serán liquidados los kilovatios (kWh) producidos, conforme a la participación en el mercado de contratos bilaterales o en la bolsa de energía.

Finalmente, el detalle de la inversión requerida para el funcionamiento del proyecto $\left(\operatorname{In} v_{n}\right)$ se estableció para cada año en los que son desarrolladas las fases de exploración, perforación y desarrollo (es decir, desde el año 0 hasta el año 7). Los rubros asociados a esta variable se deben principalmente a inversiones en preparación del campo, perforación de pozos de exploración y producción, sistemas de reinyección y construcción de la planta.

Tabla 1. Variables de entrada con distribución normal

Table 1. Input variables with normal distribution

\begin{tabular}{cccccc}
\hline Variable & Notación & Distribución & Unidades & Media & Sigma \\
\hline Temperatura de entrada & $T_{i}$ & Normal & $\mathrm{K}$ & 473,15 & 29,33 \\
\hline Precio de los contratos & $P_{\text {contratos } n}$ & Normal & COP\$/kWh & $\$ 203,03$ & $\$ 19,68$ \\
\hline Precio spot & $P_{\text {spot } n}$ & Normal & COP\$/kWh & $\$ 309,73$ & $\$ 117,01$ \\
\hline Índice de precios al productor & $I P P$ & Normal & $\%$ & $3,77 \%$ & $2,32 \%$ \\
\hline Índice de precios al consumidor & $I P C$ & Normal & $\%$ & $4,41 \%$ & $1,68 \%$ \\
\hline
\end{tabular}

Fuente: elaboración propia. Datos estimados a partir de Alfaro (2015); Mejía et al. (2014); Monsalve et al. (1998); XM (2020); XM (s.f.); Departamento Administrativo Nacional de Estadística [DANE] (2020a); DANE (2020b).

Ahora bien, para el análisis financiero del proyecto se definieron algunos parámetros técnicos y económicos necesarios para desarrollar los cálculos requeridos. Estas cifras fueron establecidas de acuerdo con el caso objeto de análisis. Los parámetros del proyecto se resumen en la Tabla 3. 
Tabla 2. Variables de entrada con distribución uniforme y triangular

Table 2. Input variables with uniform and triangular distribution

\begin{tabular}{|c|c|c|c|c|c|c|}
\hline Variable & Notación & Distribución & Unidades & Mín. & Probable & Máx. \\
\hline Contrato bilateral & $E_{\text {contratos }}$ & Uniforme & $\%$ & $70 \%$ & $80 \%$ & $100 \%$ \\
\hline Caudal & $Q$ & Triangular & $\mathrm{m}^{3} / \mathrm{seg}$ & 0,030 & 0,039 & 0,042 \\
\hline Factor de planta & $\mathrm{fp}$ & Triangular & $\%$ & $70 \%$ & $90 \%$ & $93 \%$ \\
\hline Costos O\&M fijos & $O M$ & Triangular & COP\$/kW & $\$ 202967$ & $\$ 225519$ & $\$ 257091$ \\
\hline Inversión año 0 & $\operatorname{In} v_{0}$ & Triangular & MM COP\$ & $\$ 9905$ & $\$ 11034$ & $\$ 12538$ \\
\hline Inversión año 1 & $\operatorname{In} v_{1}$ & Triangular & MM COP\$ & 9905 & $\$ 11034$ & $\$ 12538$ \\
\hline Inversión año 2 & $\operatorname{Inv} v_{2}$ & Triangular & MM COP\$ & 9905 & $\$ 11034$ & $\$ 12538$ \\
\hline Inversión año 3 & $\operatorname{Inv} v_{3}$ & Triangular & MM COP\$ & $\$ 15652$ & $\$ 17435$ & $\$ 19812$ \\
\hline Inversión año 4 & $\operatorname{In} v_{4}$ & Triangular & MM COP\$ & $\$ 20748$ & $\$ 23111$ & $\$ 26263$ \\
\hline Inversión año 5 & $\operatorname{Inv} v_{5}$ & Triangular & MM COP\$ & $\$ 38906$ & $\$ 43338$ & $\$ 49248$ \\
\hline Inversión año 6 & $\operatorname{In} v_{6}$ & Triangular & MM COP\$ & $\$ 50577$ & $\$ 56339$ & $\$ 64022$ \\
\hline Inversión año 7 & $\operatorname{In} v_{7}$ & Triangular & MM COP\$ & $\$ 40202$ & $\$ 44782$ & $\$ 50889$ \\
\hline
\end{tabular}

Tabla 3. Parámetros del proyecto

Table 3. Project parameters

\begin{tabular}{ccccc}
\hline Tipo Parámetro & Parámetro & Notación & Valor & Unidades \\
\hline Técnico & Densidad del agua & $\rho_{F}$ & 1000,00 & $\mathrm{~kg} / \mathrm{m}^{3}$ \\
\hline Técnico & Capacidad esp. calor & $c_{F}$ & 4180,00 & $\mathrm{~J} / \mathrm{kg}^{*} \mathrm{~K}$ \\
\hline Técnico & Temperatura de salida & $T_{o}$ & 393,15 & $\mathrm{~K}$ \\
\hline Técnico & No. pozos exitosos Producción & $N_{\text {pozos }}$ & 1 & $\mathrm{NA}$ \\
\hline Técnico & Degradación de la Producción & $D_{g}$ & $3 \%$ & $\%$ \\
\hline Financiero & Impuesto de renta & $T a x$ & $32 \%$ & $E A$ \\
\hline Financiero & Capital de Trabajo & $C T$ & 1 & mes sobre O\&M \\
\hline Fuente: elaboración propia. Los datos técnicos fueron estimados a partir de Knaut et al. (2013); UPME (2015).
\end{tabular}

Para el caso de la degradación anual, esta fue definida igual al 3\% (UPME, 2015), considerando que año a año la producción de energía del proyecto disminuiría en ese porcentaje, dado que la temperatura de entrada del pozo reducirá su valor a medida que la fase productiva del proyecto va avanzando.

\section{Modelo de evaluación financiera}

A continuación, se describen las componentes empleadas en el modelo de evaluación financiera para valorar la factibilidad del proyecto caso de estudio. A partir de estos elementos y las variables de entrada definidas, se realizó el cálculo del flujo de caja del proyecto y se evaluaron los criterios de decisión conocidos en la literatura como VPN y TIR, haciendo uso de simulación Monte Carlo. 


\section{Energía neta anual}

Para determinar la capacidad de la planta de energía fue necesaria la estimación de la potencia térmica $(P)$ que era capaz de generar el pozo de producción establecido para tal fin. El cálculo de esta potencia fue establecido a partir de (1), considerando las variables de entrada y parámetros requeridos.

$$
P=\left(\rho_{F} \cdot c_{F} \cdot Q \cdot\left(T_{i}-T_{o}\right)\right) / 1000
$$

- P: $\quad$ Potencia térmica $[\mathrm{kW}]$

- $\quad \rho_{F}: \quad$ Densidad del fluido $\left[\mathrm{kg} \mathrm{m}^{-3}\right]$

- $\quad c_{F}$ : Capacidad específica de calor $\left[\mathrm{J} \mathrm{kg}^{-1} \mathrm{~K}^{-1}\right]$

- Q: Caudal $\left[\mathrm{m}^{3} \mathrm{~s}^{-1}\right]$

- $\quad T_{i}$ : Temperatura de entrada $[\mathrm{K}]$

- $\quad T_{O}: \quad$ Temperatura de salida [K]

Una vez calculada la potencia térmica del pozo de producción, se aplicó (2) para determinar la energía neta anual de la planta de producción, es decir, la capacidad instalada del proyecto, la cual fue simulada a partir de diferentes escenarios, considerando que las variables temperatura de entrada $\left(T_{i}\right)$, caudal $(Q)$ y factor de planta $(f p)$ fueron definidas como variables estocásticas.

$$
E N_{n}=\left(P \cdot N_{\text {pozos }} \cdot f p \cdot H a\right) \cdot(1-D g)^{(n-1)}
$$

- $\quad E N_{n}: \quad$ Energía neta anual del año $\mathrm{n}[\mathrm{kWh}]$

- $\quad P: \quad$ Potencia térmica $[\mathrm{kW}]$

- $\quad N_{\text {pozos }}$ : Número de pozos de producción exitosos

- $\quad f p: \quad$ Factor de planta

- Ha: Número de horas en el año [8760 horas/año]

- $\quad D g$ : Degradación de la producción de energía anual (\%)

\section{Ingresos anuales}

Los ingresos de un generador de energía en Colombia se componen de la cantidad de energía que es negociada mediante contratos de largo plazo y aquellas que son tranzadas mediante la bolsa o mercado spot. De esta manera, la energía neta anual $\left(E N_{n}\right)$ es liquidada acorde a la participación de cada tipo de mercado, ya sea mediante el precio de los contratos bilaterales $\left(P_{\text {contratos }}\right)$ o el precio de bolsa $\left(P_{\text {spot }}\right)$. Los ingresos anuales del proyecto caso de estudio se encuentran expresados en (3):

$$
I_{n}=E N_{n} \cdot\left(\left(E_{\text {contratos }} \cdot P_{\text {contratos } n}\right)+\left(E_{\text {spot }} \cdot P_{\text {spot } n}\right)\right)
$$

- $\quad I_{n}: \quad$ Ingresos anuales [COP\$]

- $\quad E N_{n}: \quad$ Energía neta anual [kWh]

- $\quad E_{\text {contratos }}:$ Porcentaje de energía negociada en contratos bilaterales

- $\quad P_{\text {contratos } n}$ : Precio de los contratos bilaterales [COP\$/kWh] 
- $\quad E_{\text {spot }}: \quad$ Porcentaje de energía negociada en la bolsa $=\left(1-E_{\text {contratos }}\right)$

- $\quad P_{\text {spot } n}$ : Precio de bolsa [COP\$/kWh]

El precio de los contratos fue simulado en el primer año de operación (año 8) siguiendo una distribución normal, tal como se definió anteriormente. A partir del año 9 y hasta el final de la vida útil del proyecto, el precio de los contratos fue ajustado al índice de precios al productor (IPP). Este último, al igual que el precio spot de los kWh tranzados en este mercado, se simuló año a año de acuerdo con su distribución de probabilidad.

\section{Costos de operación y mantenimiento y capital de trabajo}

Los costos del proyecto fueron considerados a partir de la cantidad de kW de capacidad instalada de la planta al momento de su operación. Estos costos de operación y mantenimiento, una vez expresados en COP\$/kWh (de capacidad instalada en el año 8), fueron ajustados cada año de acuerdo con el índice de precios al consumidor (IPC).

La política de capital de trabajo del caso de estudio fue establecida como 1 mes sobre los costos de operación y mantenimiento. Sin embargo, es importante resaltar que, para efectos del cálculo del flujo de caja del proyecto, la cifra considerada corresponde a la variación en COP\$ de este rubro.

\section{Beneficio tributario por renta}

Los proyectos en energía renovable tienen a su favor una serie de incentivos económicos y tributarios amparados por las leyes que el gobierno colombiano ha emitido para este fin. Para efectos de este estudio, el análisis de factibilidad financiera consideró el beneficio tributario por renta que expone la Ley 1715, (2014). Este beneficio se estableció por un horizonte de 15 años, desde el año 8 hasta el 22 del proyecto, teniendo en cuenta que el valor de este rubro no debe superar el $50 \%$ de la renta líquida anual.

\section{Costo de capital del proyecto}

Se asumió que el proyecto estaría financiado en un $65 \%$ mediante deuda (D) y en un $35 \%$ a partir de recursos propios (E). Esta proporción fue definida considerando las altas sumas de inversión en capital requeridas para el funcionamiento del proyecto. El costo de la deuda $\left(K_{d}\right)$ se consideró igual al 9,05\% EA (Banco de la República, s.f.a), y el costo de patrimonio $\left(K_{e}\right)$, asumido para los inversionistas, se estableció a partir de la DTF + 7\%, siendo igual a 10,05\% EA al momento de las estimaciones (Banco de la República, s.f.b). Dada la estructura de capital, se calculó el costo de capital del proyecto (WACC) a través de (4), el cual se estimó igual a 7,52\% EA:

$$
W A C C=K_{e} \cdot\left(\frac{E}{D+E}\right)+K_{d} \cdot(1-\operatorname{Tax}) \cdot\left(\frac{D}{D+E}\right)=7,52 \% E A
$$




\section{Valoración de opciones reales}

Posterior al análisis de factibilidad financiera del proyecto mediante los métodos tradicionales, se aplicó enfoque de opciones reales para valorar la flexibilidad de los inversionistas en el proyecto caso de estudio.

Este análisis fue realizado a partir de la aplicación de una opción de abandono compuesta haciendo uso del método de árbol binomial, este tipo de opción real fue evaluada considerando que los proyectos en energía geotérmica se caracterizan por tener largar fases de inversión, sobre las cuales los inversionistas adquieren información acerca de las especificaciones técnicas del proyecto y las características de los recursos. A partir de estos datos, los inversionistas pueden decidir continuar con el proyecto o abandonarlo si la información obtenida en la etapa previa a su próxima inversión no resulta favorable.

Bajo el supuesto que los flujos del proyecto se comportan con una distribución logarítmica-normal, la volatilidad del proyecto se estimó siguiendo el método Market Asset Disclaimer-MAD- (Copeland y Antikarov, 2001), a partir de (5).

$$
z=\ln \left(\frac{V P_{1}+F C F_{1}}{V P_{0}}\right)
$$

Donde $V P_{1}$ es valor presente en el momento 1 de los flujos del proyecto, $F C F_{1}$ el flujo de ese año y $V P_{0}$ el valor presente de los flujos de caja del proyecto, ubicados un año previo a la operación. El numerador de esta expresión es simulado mientras que $V P_{0}$ permanece constante para efectos de este cálculo.

Las ecuaciones (6) - (8) representan el modelo de árboles binomiales, donde el valor del activo subyacente tiene la posibilidad de incrementarse $(\mathrm{u}$ ) con una probabilidad de $\mathrm{p}$, o disminuir (d) con probabilidad (1-p) en cualquier periodo de tiempo.

$$
\begin{gathered}
u=e^{\sigma \sqrt{T}} \\
d=\frac{1}{u} \\
p=\frac{(1+r f)-d}{u-d}
\end{gathered}
$$

Donde $T$ es la división de tiempo y rf la tasa libre de riesgo. Para este caso de estudio, rf fue considerado como el rendimiento de un título del tesoro emitido por el gobierno colombiano (TES) con vencimiento a 16 años.

Por su parte, el valor de la opción fue calculada aplicando (9), la cual considera las inversiones requeridas en cada año $(K)$. 


$$
C=\operatorname{Max}\left(\left(\frac{1}{1+r f} *\left[\left(p * C_{u}\right)+\left((1-p) * C_{d}\right)\right]\right)-K ; 0\right)
$$

De esta manera fue valorada la flexibilidad de los inversionistas del proyecto, los cuales podrían tomar la decisión de no invertir o abandonar el proyecto, en cada uno de los nodos del árbol.

\section{RESULTADOS}

Los resultados producto de esta investigación fueron obtenidos mediante la simulación de 100000 escenarios usando la herramienta @Risk. La Tabla 4 muestra el detalle del flujo de caja libre para los primeros 8 años del proyecto. Esta información es complementada en el Anexo, en el cual se encuentra el flujo de caja libre desde el año 9 hasta el año 22 del proyecto.

Tabla 4. Flujo de caja libre del proyecto

Table 4. Free cash flow of the project

\begin{tabular}{|c|c|c|c|c|c|c|c|c|c|}
\hline & 0 & 1 & 2 & 3 & 4 & 5 & 6 & 7 & 8 \\
\hline Ingresos anuales & & & & & & & & & 23004 \\
\hline Ingresos por contratos & & & & & & & & & 16653 \\
\hline$\%$ Contratos bilaterales & & & & & & & & & $80 \%$ \\
\hline Precio de los contratos [COP/kWh] & & & & & & & & & 203,03 \\
\hline Ingresos por mercado spot & & & & & & & & & 6351 \\
\hline \% Energía en bolsa & & & & & & & & & $20 \%$ \\
\hline Precio spot [COP\$/kWh] & & & & & & & & & 309,73 \\
\hline Costos O\&M fijos & & & & & & & & & 288,06 \\
\hline Costos O\&M fijos [COPS/kW-ai & iño] & & & & & & & & 22151 \\
\hline Costos O\&M fijos [COP\$/kWh] & & & & & & & & & 2,81 \\
\hline Depreciación & & & & & & & & & 10905 \\
\hline EBIT & & & & & & & & & 11811 \\
\hline Impuestos (32\%) & & & & & & & & & 3779 \\
\hline NOPAT & & & & & & & & & 8031 \\
\hline CAPEX & 11034 & 11034 & 11034 & 17435 & 23111 & 43338 & 56339 & 44782 & 0 \\
\hline Variación de CT & & & & & & & & & 24,01 \\
\hline Capital de Trabajo & & & & & & & & & 24,01 \\
\hline Valor a deducir Ley 1715 & & & & & & & & & 1890 \\
\hline Beneficio tributario Ley 1715 & & & & & & & & & 7270 \\
\hline FLUJO DE CAJA LIBRE & -11034 & -11034 & -11034 & -17435 & -23111 & -43338 & -56339 & -44782 & 20802 \\
\hline
\end{tabular}

Adicionalmente, la Figura 1 representa el flujo de caja libre del caso de estudio para uno de los escenarios simulados, donde se aprecia que los primeros años del proyecto requieren altas inversiones (Año 0 - Año 7) y su flujo de caja toma valores positivos a partir del octavo año. 


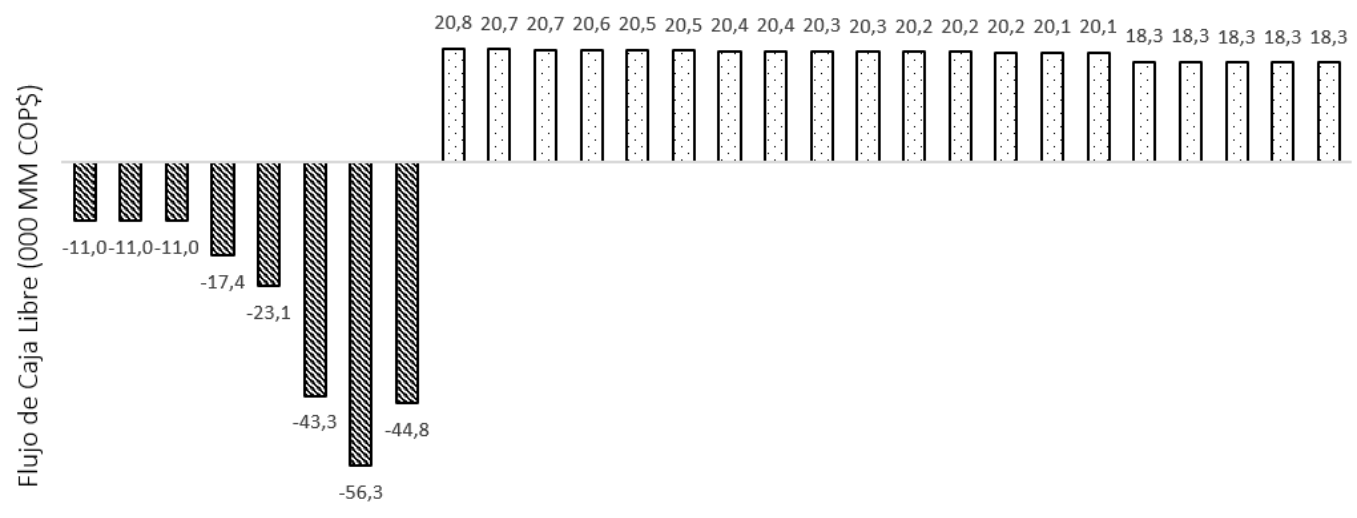

Año

Figura 1. Flujo de caja libre del proyecto

Figure 1. Free cash flow of the project Fuente: elaboración propia.

\section{Análisis de factibilidad financiera}

Haciendo uso del método clásico de valoración, el VPN del proyecto fue igual a -\$47542 MM COP, correspondiente a la media de las simulaciones realizadas vía Monte Carlo. Como se puede observar en la Figura 2, el proyecto tuvo un VPN menor a cero en la mayor parte de los escenarios obtenidos, clasificando como inviable este proyecto de inversión.

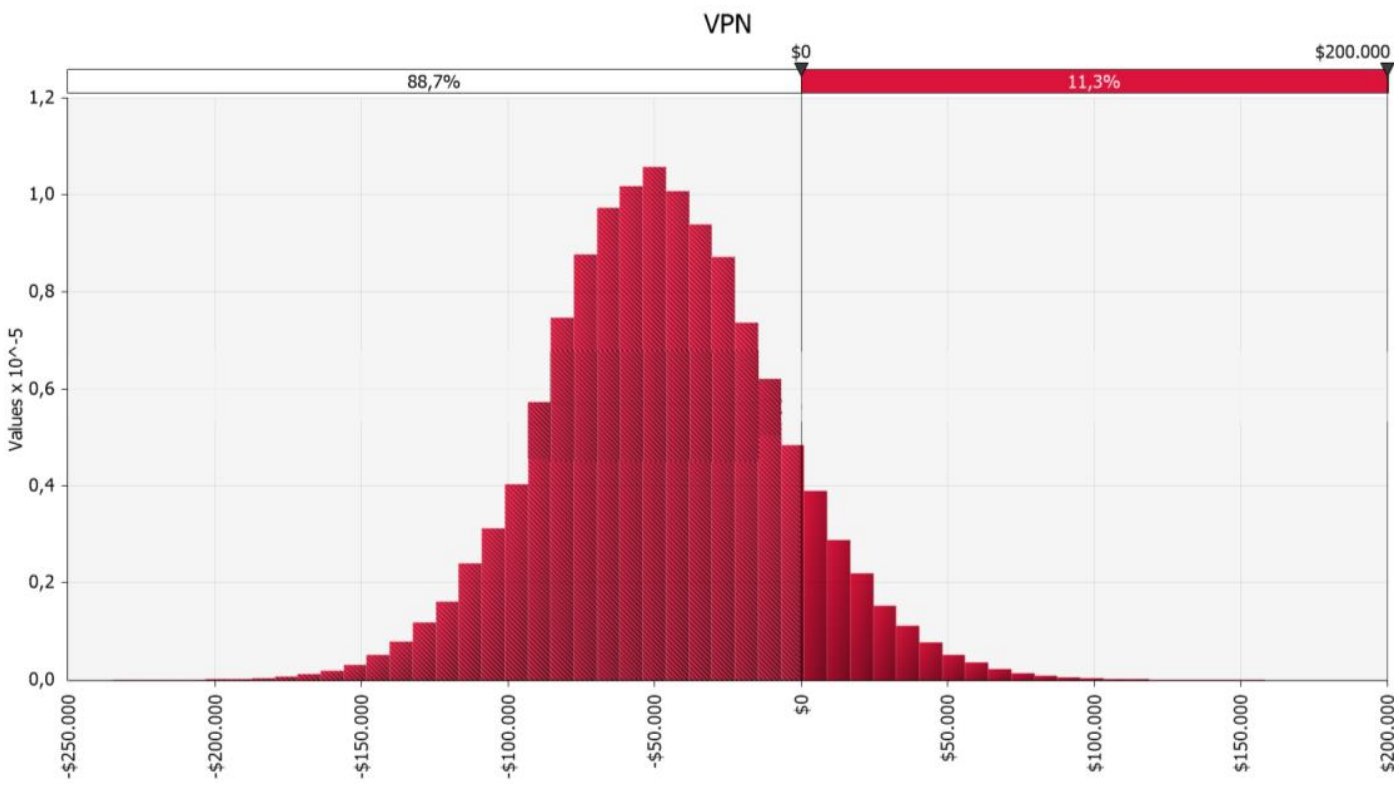

Figura 2. VPN del proyecto

Figure 2. NPV of the project

Fuente: elaboración propia usando el software @Risk. 
Aplicando el criterio conocido como tasa interna de retorno, los resultados obtenidos a partir de las simulaciones indicaron que el $88,4 \%$ de las veces el proyecto tuvo una TIR inferior al WACC $(7,52 \%)$. Es decir, en la mayor parte de los escenarios simulados el criterio de decisión asociado clasifica el proyecto como no factible, al no retornar la mínima rentabilidad esperada. En otras palabras, haciendo uso de dos de los criterios tradicionales más usados en la literatura, el proyecto caso de estudio no genera valor económico para los inversionistas.

A partir de la información obtenida mediante el software de simulación, la Figura 3 presenta la correlación existente entre algunas de las variables de entrada definidas y el VPN del proyecto.

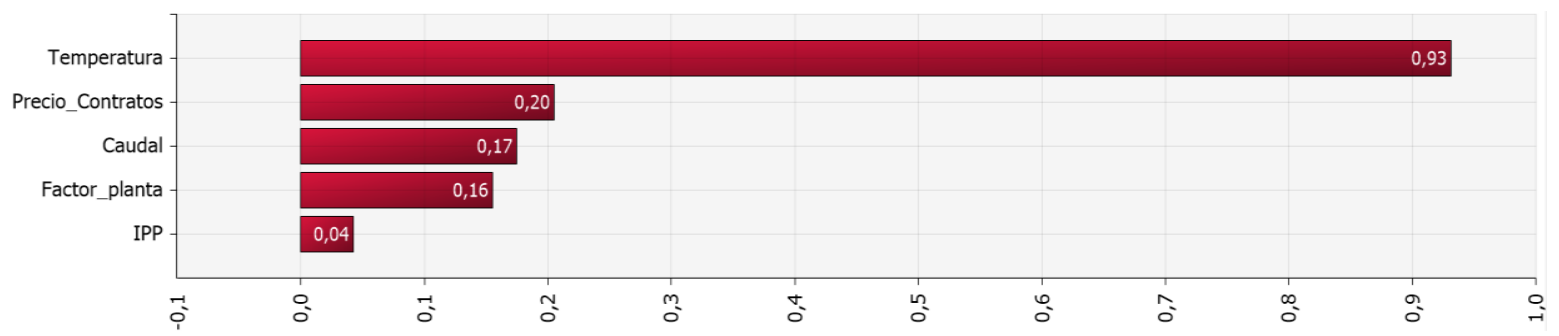

Figura 3. Correlación de las variables con el VPN

Figure 3. Correlation of the variables with the NPV

Fuente: elaboración propia usando el software @Risk.

Como se puede observar, 2 de las 3 variables que tienen mayor correlación con el VPN del proyecto son variables asociadas a los recursos geotérmicos del lugar donde se desarrolla el proyecto: la temperatura de entrada $\left(T_{i}\right)$ y el caudal $(Q)$, siendo $T_{i}$ la variable con mayor correlación, seguida por el precio de los contratos bilaterales y el caudal del recurso. Para el caso de la temperatura de entrada, esta variable registra una correlación directa y casi perfecta, indicando una alta dependencia entre la factibilidad del proyecto y el valor de la temperatura del pozo geotérmico. Esto también se vio reflejado en el análisis de sensibilidad, el cual indicó que esta variable es la más influyente en el VPN del proyecto al ser única, cuya variación tiene como efecto directo un VPN positivo igual a \$16 611 MM COP.

\section{Análisis de Opciones Reales}

Mediante la aplicación de (5), y haciendo uso del software de simulación, se obtuvo que la volatilidad del proyecto $(\sigma)$ fue del $44,96 \%$, la cual corresponde a la desviación estándar de los rendimientos del proyecto (z). Esta alta volatilidad responde a la incertidumbre característica de estos proyectos, que para este caso de estudio está asociada a los recursos geotérmicos y variables del mercado de energía. Posteriormente se realizó la construcción del árbol binomial del activo subyacente y de la opción con un detalle anual. Los parámetros utilizados para la valoración de la opción y los valores obtenidos se presentan en la Tabla 5. 
Tabla 5. Parámetros de la valoración de la opción

Table 5. Option valuation parameters

\begin{tabular}{cccc}
\hline Parámetros & Símbolo & Valor & Detalle \\
\hline Precio de ejercicio (MM COP) & $\mathrm{K}$ & Inv & Desembolsos requeridos cada año \\
\hline Valor del subyacente (MM COP) & $\mathrm{S}$ & $\$ 109998$ & VP de los flujos del proyecto \\
\hline Volatilidad & $\sigma$ & $44,96 \%$ & Desv. estándar del proyecto \\
\hline Tasa libre de riesgo & $\mathrm{rf}$ & $6,272 \%$ & Banco de la República (s.f.c) \\
\hline Factor de subida & $\mathrm{u}$ & 1,568 & Ecuación (6) \\
\hline Factor de caída & $\mathrm{d}$ & 0,638 & Ecuación (7) \\
\hline Probabilidad de alza & $\mathrm{p}$ & $45,69 \%$ & Ecuación (8) \\
\hline Probabilidad de baja & $(1-\mathrm{p})$ & $54,31 \%$ & \\
\hline & Fuente: elaboración propia.
\end{tabular}

La construcción del árbol del activo subyacente, parte del valor del proyecto a hoy (\$109998 COP MM), cifra que fue actualizada en cada una de las ramas a partir de los multiplicadores de ascenso (u) y descenso (d). Como se puede observar en la Figura 4, el árbol del activo subyacente se construyó hasta el año número 7, periodo en el cual concluyen las fases de inversión. Es en este año que el valor del activo subyacente registró los valores más altos y bajos que puede tomar el valor del proyecto geotérmico.

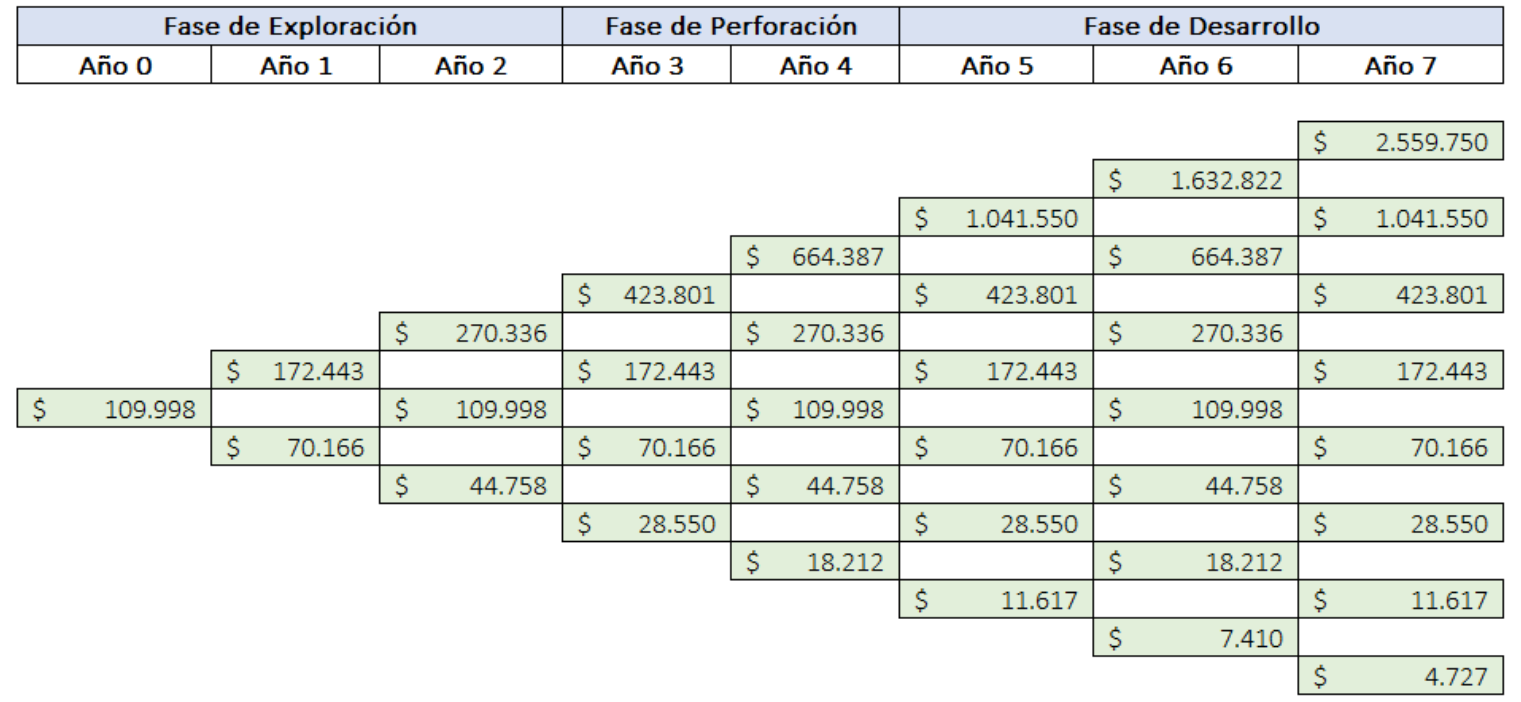

Figura 4. Árbol binomial del activo subyacente

Figure 4. Binomial tree of the underlying asset

Fuente: elaboración propia.

A partir del árbol binomial de la opción se obtuvo el valor del proyecto con flexibilidad, como se puede observar en la Figura 5. Este resultado fue obtenido considerando las inversiones requeridas en cada una de las fases de inversión y aplicando (9). Así pues, el proyecto adquiere un valor de \$7149 MM COP al ser analizado mediante el enfoque de opciones reales, indicando que invertir en este proyecto es viable desde el punto de vista financiero. 


\begin{tabular}{|l|c|c|c|c|c|c|c|}
\hline \multicolumn{3}{|c|}{ Fase de Exploración } & \multicolumn{3}{c|}{ Fase de Perforación } & \multicolumn{3}{c|}{ Fase de Desarrollo } \\
\hline Año 0 & Año 1 & Año 2 & Año 3 & Año 4 & Año 5 & Año 6 & Año \\
\hline
\end{tabular}

$\begin{array}{llllllllllllllll}\$ & 11.034 & \$ & 11.034 & \$ & 11.034 & \$ & 17.435 & \$ & 23.111 & \$ & 43.338 & \$ & 56.339 & \$ & 44.782\end{array}$

\begin{tabular}{|c|c|c|c|c|c|c|c|c|c|c|c|c|c|c|c|}
\hline & & & & & & & & & & & & & & $\$$ & 2.514 .968 \\
\hline & & & & & & & & & & & & $\$$ & 1.534 .343 & & \\
\hline & & & & & & & & & & $\$$ & 905.545 & & & $\$$ & 996.767 \\
\hline & & & & & & & & $\$$ & 513.298 & & & $\$$ & 565.908 & & \\
\hline & & & & & & $\$$ & 264.195 & & & $\$$ & 287.797 & & & $\$$ & 379.019 \\
\hline & & & & $\$$ & 119.846 & & & $\$$ & 119.247 & & & $\$$ & 171.857 & & \\
\hline & & $\$$ & 42.289 & & & $\$$ & 33.835 & & & $\$$ & 36.438 & & & $\$$ & 127.660 \\
\hline$\$$ & 7.149 & & & $\$$ & 3.514 & & & $\$$ & - & & & $\$$ & 11.520 & & \\
\hline & & $\$$ & - & & & $\$$ & - & & & $\$$ & - & & & $\$$ & 25.384 \\
\hline & & & & $\$$ & - & & & $\$$ & - & & & $\$$ & - & & \\
\hline & & & & & & $\$$ & - & & & $\$$ & - & & & $\$$ & - \\
\hline & & & & & & & & $\$$ & - & & & $\$$ & - & & \\
\hline & & & & & & & & & & $\$$ & - & & & $\$$ & - \\
\hline & & & & & & & & & & & & $\$$ & - & & \\
\hline & & & & & & & & & & & & & & $\$$ & - \\
\hline
\end{tabular}

Figura 5. Árbol binomial de la opción

Figure 5. Binomial tree of the option Fuente: elaboración propia.

Las casillas de color gris representan aquellos escenarios en los que el proyecto sería abandonado por los inversionistas al no ser atractivo invertir en la siguiente fase si el valor del activo subyacente no superaba la inversión requerida en ese momento. Para este caso en particular, el inversionista invertiría el capital requerido en el año 1 , y en el caso de que el proyecto no resultara favorable para él, se retiraría antes de continuar invirtiendo las altas sumas de capital que demandan las fases posteriores.

La Tabla 6 resume los resultados obtenidos de la valoración del proyecto caso de estudio haciendo uso del método clásico VPN versus la valoración mediante opciones reales.

Tabla 6. Valor del proyecto

Table 6. Project value

\begin{tabular}{cc}
\hline Valor del proyecto & MM COP \\
\hline VPN sin flexibilidad & $-\$ 47542$ \\
\hline VPN con flexibilidad & $\$ 7149$ \\
\hline Valor opcionalidad & $\$ 54691$ \\
\hline \multicolumn{2}{c}{ Fuente: elaboración propia. }
\end{tabular}

La sensibilidad del valor del proyecto ante escenarios de mayor incertidumbre se presenta en la Tabla 7. Como se puede observar, el valor del proyecto mediante el enfoque de opciones reales es cada vez mayor a medida que la incertidumbre (representada por la desviación estándar del proyecto) aumenta. 
Tabla 7. Sensibilidad del valor del proyecto a la volatilidad

Table 7. Sensitivity of the project value to volatility

\begin{tabular}{ccc}
\hline Desviación estándar & VPN con flexibilidad (MM COP) & Valor Opcionalidad (MM COP) \\
\hline $50 \%$ & $\$ 11248$ & $\$ 58790$ \\
\hline $60 \%$ & $\$ 19007$ & $\$ 66549$ \\
\hline $70 \%$ & $\$ 26546$ & $\$ 74088$ \\
\hline $80 \%$ & $\$ 33496$ & $\$ 81038$ \\
\hline
\end{tabular}

\section{DISCUSIÓN}

Los proyectos en energía, en especial aquellos de naturaleza renovable, se caracterizan por tener asociadas a sus variables de entrada altas incertidumbres que se reflejan en los resultados obtenidos en los flujos de caja de los proyectos. Bajo esta premisa, el valor agregado de esta investigación está en considerar elementos de valoración adicionales a los evaluados en los métodos clásicos de la literatura al involucrar las opciones reales como herramienta para valorar las flexibilidades de los inversionistas a lo largo de la vida útil del proyecto.

Además de caracterizarse por los altos costos de inversión, los proyectos en energía geotérmica tienen asociado un gran riesgo e incertidumbre, aspectos que deben ser considerados al momento de evaluar la viabilidad económica de cada proyecto, pues tienen una gran influencia en su factibilidad y deben ser tomados en cuenta antes de realizar grandes inversiones (Knaut et al., 2013). En concordancia con investigaciones previas (Ehyaei et al., 2020; Schulz et al., 2010), desde el punto de vista técnico existen diversas variables que afectan el rendimiento de un sistema geotérmico, entre ellas, la temperatura de entrada del fluido y su caudal. Los resultados obtenidos a partir de este estudio indicaron que estas variables, en especial la temperatura de entrada, tienen una alta incidencia en la factibilidad financiera de proyectos de este tipo. La temperatura del fluido geotérmico está estrechamente relacionada con la profundidad del pozo. Por lo tanto, la optimización de estas variables genera, sin duda, efectos positivos en el proyecto en términos energéticos y termodinámicos. De este modo, una de las barreras que se atribuyen para la explotación de la energía geotérmica está relacionada con el alto riesgo por la incertidumbre en la fase de exploración del proyecto (Santoyo y Barragán-Reyes, 2010), ya que muchos datos geológicos como el tipo de recurso, la temperatura, el gradiente geotérmico y la tasa de producción potencial no pueden ser determinados con seguridad antes de la perforación de pozos que lo confirmen (Compernolle et al., 2019; Zhang et al., 2019).

Los factores de riesgo e incertidumbre, como base de esta investigación, fueron evaluados con el fin de dar un resultado más real y ajustado a la realidad de esta clase de proyectos. El análisis de opciones realizado permitió valorar la oportunidad que tienen los inversionistas de abandonar o no el proyecto al final de cada uno de los años de inversión. En efecto, los resultados indican que el proyecto sí es factible, lo cual contrasta totalmente con el criterio emitido por el VPN tradicional y la TIR, siendo evidente que, mediante la aplicación de los métodos clásicos, el valor del proyecto se estaría subestimando. Un resultado similar fue obtenido por Bilqist et al. (2018), quienes hicieron uso de 3 criterios diferentes VPN, valor presente neto esperado (ENPV, por sus siglas en inglés) y opciones reales para evaluar la viabilidad de un proyecto de energía geotérmica en Indonesia. Este estudio 
encontró que mediante el enfoque de valoración de opciones reales el proyecto evaluado era más factible en comparación con los resultados de los otros dos criterios, en los cuales no fueron capturados ciertos elementos que agregaban valor al proyecto.

De acuerdo con Compernolle et al. (2019), además de los factores geológicos de los proyectos geotérmicos se derivan grandes incertidumbres en el campo tecnológico y económico. Haciendo uso del conocido método de simulación Monte Carlo, los autores desarrollaron un modelo geoeconómico que permitió evaluar diferentes medidas gubernamentales como la devolución de impuestos y una prima por cada milivatio-hora ( $\mathrm{mWh}$ ) producido. Además, el análisis incorporó las opciones reales para valorar la opción de abandono después de que la primera perforación del yacimiento geotérmico ha sido realizada.

De este modo, las estimaciones incorrectas de los riesgos vienen acompañadas de las grandes incertidumbres que afectan los proyectos en energía geotérmica, especialmente en lo que concierne a factores geológicos, siendo la cuantificación de los riesgos implicados en la etapa de exploración un aspecto fundamental para el desarrollo de esta clase de proyectos y las evaluaciones incorrectas de los riesgos pueden ser evitadas con bases de datos que den soporte a los cálculos requeridos para estos estudios (Ganz, 2015).

Para futuros trabajos, las investigaciones deberían estar enfocadas en maximizar la potencia de la instalación geotérmica a partir diferentes parámetros técnicos, minimizando los costos de operación. Adicionalmente se propone como tema de investigación analizar el efecto económico del uso eficiente del fluido geotérmico para la producción de energía, debido a la reducción de los costos asociados a los impuestos por la utilización de estos recursos.

\section{CONCLUSIONES}

Este estudio presenta un marco de valoración de proyectos de energía, en el cual se demuestra que el análisis de factibilidad financiera debe considerar características acordes al entorno en el cual se desarrolla el proyecto, lo cual involucra la toma de decisiones de los inversionistas, la incertidumbre de las variables y los riesgos inherentes.

A partir de esta investigación se pudo observar que las características geológicas del lugar donde se lleva a cabo el proyecto geotérmico tienen un gran impacto sobre la factibilidad del proyecto. Un aumento en recursos como la temperatura pueden generar que el valor del proyecto mediante el VPN tradicional sea atractivo para los inversionistas. De esta manera, las primeras fases del proyecto, en las cuales se identifica el tipo de recurso geotérmico y la calidad de este, son de vital importancia para el desarrollo de las fases posteriores y la continuidad del proyecto. De hecho, un incremento en la temperatura del fluido geotérmico conlleva a un incremento de la eficiencia térmica de la planta. Es de esperarse que, a medida que aumente la profundidad del pozo, haya un aumento en la temperatura del fluido geotérmico y, por lo tanto, en la potencia de salida.

Para este análisis, la valoración financiera a través del método clásico subestima el valor del proyecto en \$54 691 MM, al no tener en cuenta la flexibilidad operativa a lo largo de sus fases de inversión, indicando que un proyecto geotérmico en Colombia no sería atractivo para los inversionistas. Así, los 
criterios de decisión tradicionales consideran un escenario estático, asumiendo valores certeros e irreversibles, donde los flujos de caja del proyecto no tendrán estados más o menos favorables de los previstos en la valoración inicial. Sin embargo, al aplicar las opciones reales como método de valoración financiera, el proyecto es considerado viable financieramente, ya que permite evaluar los escenarios de decisión sobre los cuales puede decidir el inversionista a medida que adquiere más información sobre el tipo de recurso geotérmico y las condiciones del mercado.

Finalmente, la aplicación de las opciones reales, como método de valoración para este estudio, demuestra que la incertidumbre es un factor que se debe tener en cuenta al momento de evaluar la viabilidad financiera de proyectos, en especial los pertenecientes al sector de la energía. Lo anterior, dado que es un parámetro que agrega valor al proyecto, al considerar diferentes escenarios y flexibilidades, características que se ajustan a la realidad de los proyectos de inversión.

\section{CONFLICTOS DE INTERÉS}

Los autores declaran que no presentan conflictos de interés financiero, profesional o personal que pueda influir de forma inapropiada en los resultados obtenidos o las interpretaciones propuestas.

\section{CONTRIBUCIÓN DE AUTORES}

Para el desarrollo de este proyecto todos los autores han realizado una contribución significativa especificada a continuación:

Yessenia Martínez-Ruiz: escritura del artículo y desarrollo de la investigación.

Diego Fernando Manotas-Duque: tutoría de la investigación, conceptualización, revisión y redacción final de manuscrito.

Howard Ramírez-Malule: co - tutoría de la investigación, revisión y redacción final del manuscrito.

\section{REFERENCIAS}

Abadie, L. M. (2009). Valuation of long-term investments in energy assets under uncertainty. Energies, v. 2, n. 3, 738-768. https://doi.org/10.3390/en20300738

Agaton, C. (2017). Real Options Analysis of Renewable Energy Investment Scenarios in the Philippines. Renewable Energy and Sustainable Development, v. 3, n. 3, 284-292. https://doi.org/10.21622/resd.2017.03.3.284

Aguilera Bustos, J. P., Herrera Román, C. P. (2017). Evaluación De Factibilidad Técnico-Financiera del Desarrollo Inicial de un Campo Geotérmico con Planta a Bocapozo [Diplomado, Universidad de El Salvador]. URL 
Alfaro, C. (2015). Improvement of Perception of the Geothermal Energy as a Potential Source of Electrical Energy in Colombia, Country Update. URL

Banco de la República. (s.f.a). Tasas de colocación consolidadas. $\underline{\text { URL }}$

Banco de la República. (s.f.b). Tasas de captación semanales y mensuales. $\underline{\text { URL }}$

Banco de la República. (s.f.c). TES en pesos. URL

Bandoc, G., Degeratu, M., Florescu, A. M., Dragomir, E. (2013). Variance analysis of wind characteristics for energy conversion. Journal of Environmental Protection and Ecology, $\mathrm{v}$. 14, n. 4, 1760-1768. URL

Bazmi, A. A., Zahedi, G. (2011). Sustainable energy systems: Role of optimization modeling techniques in power generation and supply - A review. Renewable and Sustainable Energy Reviews, v. 15, n. 8, 3480-3500. https://doi.org/10.1016/j.rser.2011.05.003

Bilqist, R. A., Dachyar, M., Farizal, F. (2018, 18 de octubre). Project Valuation in the Geothermal Power Plant Project: A Comparison of Expected Net Present Value and Static Net Present Value Approaches. International Conference On Multidisciplinary Academic (ICMA) 2018, Indonesia. https://doi.org/10.2139/ssrn.3248120

Brach, M. A. (2003). Real Options in practice. John Wiley \& Sons.

Compernolle, T., Welkenhuysen, K., Petitclerc, E., Maes, D., Piessens, K. (2019). The impact of policy measures on profitability and risk in geothermal energy investments. Energy Economics, v. 84, 104524. https://doi.org/10.1016/i.eneco.2019.104524

Copeland, T., Antikarov, V. (2001). Real options: A practitioner's guide. Texere.

Córdoba Cárdenas, C., Flórez Alzate, D., Arango Uribe, D. (2019). Colombia, ¿cerca de la ola renovable? $\underline{\mathrm{URL}}$

Coviello, M. F. (2000). Estudio para la Evaluación del Entorno del Proyecto Geotérmico Binacional «Tufiño-Chiles-Cerro Negro». Comisión Económica para América Latina y el Caribe. URL

Damodaran, A. (2005). The Promise and Peril of Real Options. En NYU Working Paper, 1-75. URL

Daneshvar, M., Eskandari, H., Sirous, A. B., Esmaeilzadeh, R. (2021). A novel techno-economic risk-averse strategy for optimal scheduling of renewable-based industrial microgrid. Sustainable Cities and Society, v. 70, 102879. https://doi.org/10.1016/j.scs.2021.102879

Departamento Administrativo Nacional de Estadística. (2020a). Índice de Precios al Consumidor (IPC). Índices - Series de empalme. $\underline{U R L}$ 
Análisis de opciones reales para la valoración financiera de proyectos de energía geotérmica en Colombia

Departamento Administrativo Nacional de Estadística. (2020b). Índice de precios del productor (IPP). Series históricas Índices de Precios del Productor. URL

Ehyaei, M. A., Ahmadi, A., Rosen, M. A., Davarpanah, A. (2020). Thermodynamic optimization of a geothermal power plant with a genetic algorithm in two stages. Processes, v. 8, n. 10, 116. https://doi.org/10.3390/pr8101277

Fallah, M., Akbarpour Ghiasi, R., Mokarram, N. H. (2018). A comprehensive comparison among different types of geothermal plants from exergy and thermoeconomic points of view. Thermal Science and Engineering Progress, v. 5, 15-24.

https://doi.org/10.1016/i.tsep.2017.10.017

Fernandes, B., Cunha, J., Ferreira, P. (2011). The use of real options approach in energy sector investments. Renewable and Sustainable Energy Reviews, v. 15, n. 9, 4491-4497.

https://doi.org/10.1016/j.rser.2011.07.102

Ganz, B. (2015). Evaluation of Expert Reports to Quantify the Exploration Risk for Geothermal Projects in Germany. Energy Procedia, v. 76, 341-350.

https://doi.org/10.1016/i.egypro.2015.07.839

García Espinoza, P. A. (2016). Aplicación de Opciones Reales en la Valoración Financiera de un Campo Petrolero [Tesis de maestría, Universidad Externado de Colombia]. $\underline{\text { URL }}$

Gavrikova, E., Burda, Y., Gavrikov, V., Sharafutdinov, R., Volkova, I., Rubleva, M., Polosukhina, D. (2019). Clean energy sources: insights from Russia. Resources, v. 8, n. 2, 84. https://doi.org/10.3390/resources8020084

Gonima Mena, V. (2017). Avances recientes en el uso de opciones reales en la evaluación de proyectos de generación de energía con fuentes alternativas. Maestría en ingeniería [Tesis de maestría, Universidad Nacional de Colombia].

https://repositorio.unal.edu.co/handle/unal/58716

Isaza Cuervo, F. (2016). Valoración de fuentes renovables no convencionales de generación de electricidad: un enfoque desde las opciones reales. Cuadernos de Administración, v. 28, n. 51, 45-64. https://doi.org/10.11144/javeriana.cao28-51.vfrc

Isaza Cuervo, F., Botero Botero, S. (2014). Aplicación de las Opciones Reales en la Toma de Decisiones en los Mercados de Electricidad. Estudios Gerenciales, v. 30, n. 133, 397-407. https://doi.org/10.1016/j.estger.2014.06.003

Ju, L., Tan, Q., Lu, Y., Tan, Z., Zhang, Y., Tan, Q. (2019). A CVaR-robust-based multi-objective optimization model and three-stage solution algorithm for a virtual power plant considering uncertainties and carbon emission allowances. International Journal of Electrical Power \& Energy Systems, v. 107, 628-643.

https://doi.org/10.1016/j.ijepes.2018.12.012 
Kim, K., Park, H., Kim, H. (2017). Real options analysis for renewable energy investment decisions in developing countries. Renewable and Sustainable Energy Reviews, v. 75, 918-926. https://doi.org/10.1016/i.rser.2016.11.073

Knaut, A., Madlener, R., Rosen, C., Vogt C. (2013). Effects of Temperature Uncertainty on the Valuation of Geothermal Project: A Real Options Approach. FCN Working Paper, n. 11, 146. https://doi.org/10.2139/ssrn.2329563

Lesmana, A., Winofa, N. C., Pratama, H. B., Ashat, A., Saptadji, N. M. (2020). Preliminary Financial Modelling with Probabilistic Approach for Geothermal Development Project in Indonesia. IOP Conference Series: Earth and Environmental Science, v. 417, 012024. https://doi.org/10.1088/1755-1315/417/1/012024

Ley 1715 de 2014. (2014). Por medio de la cual se regula la integración de las energías renovables no convencionales al sistema energético nacional. 13 de mayo de 2014.

Li, Y., Wang, J., Han, Y., Zhao, Q., Fang, X., Cao, Z. (2020). Robust and opportunistic scheduling of district integrated natural gas and power system with high wind power penetration considering demand flexibility and compressed air energy storage. Journal of Cleaner Production, v. 256, 120456. https://doi.org/10.1016/j.jclepro.2020.120456

Li, Y., Yang, W., Tian, L., Yang, J. (2018). An evaluation of investment in a PV power generation project in the gobi desert using a real options model. Energies, v. 11, n. 1, 257. https://doi.org/10.3390/en11010257

Liu, L., Zhang, M., Zhao, Z. (2019). The application of real option to renewable energy investment: A review. Energy Procedia, v. 158, 3494-3499. https://doi.org/10.1016/i.egypro.2019.01.921

Manotas Duque, D. F., Manyoma, P. C. (2001). La Evaluación de proyectos de inversión mediante opciones reales: Aspectos conceptuales. Ingenieria y Competitividad, v. 3, n. 1, 7-18. https://doi.org/10.25100/iyc.v3i1.2332

Martínez-Ruiz, Y., Manotas-Duque, D. F., Ramírez-Malule, H. (2020). Evaluation of investment projects in photovoltaic solar energy using the DNPV methodology. International Journal of Energy Economics and Policy, v. 11, n. 1, 180-185. https://doi.org/10.32479/ijeep.10577

Mauboussin, M. J. (1999). Get real: Using Real Options in Security Analysis. URL

Mejía, E., Rayo, L., Méndez, J., Echeverri, J. (2014). Geothermal development in Colombia. URL

Monsalve, M. L., Rodríguez, G. I., Mendez, R. A., Bernal, N. F. (1998). Geology of the Well Nereidas 1, Nevado Del Ruiz Volcano, Colombia. Geothermal Resources Council Transactions, v. 22, 263-267. URL 
Mun, J. (2002). Real Options Analysis: Tools and Techniques for Valuing Strategic Investments and Decisions. John Wiley \& Sons.

Resolución 030 de 2018. Por la Cual se regulan las actividades de autogeneración a pequeña escala y de generación distribuida en el sistema interconectado nacional. (2018, 01 de marzo). Ministerio de Minas y Energía. D.O. No. 50.522.

Rocha, L. C. S., Aquila, G., Junior, P. R., de Paiva, A. P., de Oliveira Pamplona, E., Balestrassi, P. P. (2018). A stochastic economic viability analysis of residential wind power generation in Brazil. Renewable and Sustainable Energy Reviews, v. 90, 412-419.

https://doi.org/10.1016/j.rser.2018.03.078

Santos, L., Soares, I., Mendes, C., Ferreira, P. (2014). Real Options versus Traditional Methods to assess Renewable Energy Projects. Renewable Energy, v. 68, 588-594. https://doi.org/10.1016/i.renene.2014.01.038

Santoyo, E., Barragán-Reyes, R. M. (2010). Energía Geotérmica. Revista de la Academia Mexicana de Ciencias, v. 61, n. 2, 40-51. URL

Schulz, R., Pester, S., Schellschmidt, R., Thomas, R. (2010). Quantification of Exploration Risks as Basis for Insurance Contracts. Proceedings World Geothermal Congress, 25-29. URL

Unidad de Planeación Minero Energética. (2015). Integración de las energías renovables no convencionales en Colombia. $\underline{\mathrm{URL}}$

XM. (2020). Estadísticas de registro de contratos de largo plazo mensual. Año 2020. URL

XM. (s.f.). Precio de bolsa y escasez. $\underline{\mathrm{URL}}$

Zhang, M., Liu, L., Wang, Q., Zhou, D. (2020). Valuing investment decisions of renewable energy projects considering changing volatility. Energy Economics, v. 92, 104954. https://doi.org/10.1016/j.eneco.2020.104954

Zhang, Q., Chen, S., Tan, Z., Zhang, T., Mclellan, B. (2019). Investment strategy of hydrothermal geothermal heating in China under policy, technology and geology uncertainties. Journal of Cleaner Production, v. 207, 17-29. https://doi.org/10.1016/j.jclepro.2018.09.240 


\section{ANEXO}

Tabla 8. Flujo de caja libre del proyecto

Table 8. Project free cash flow

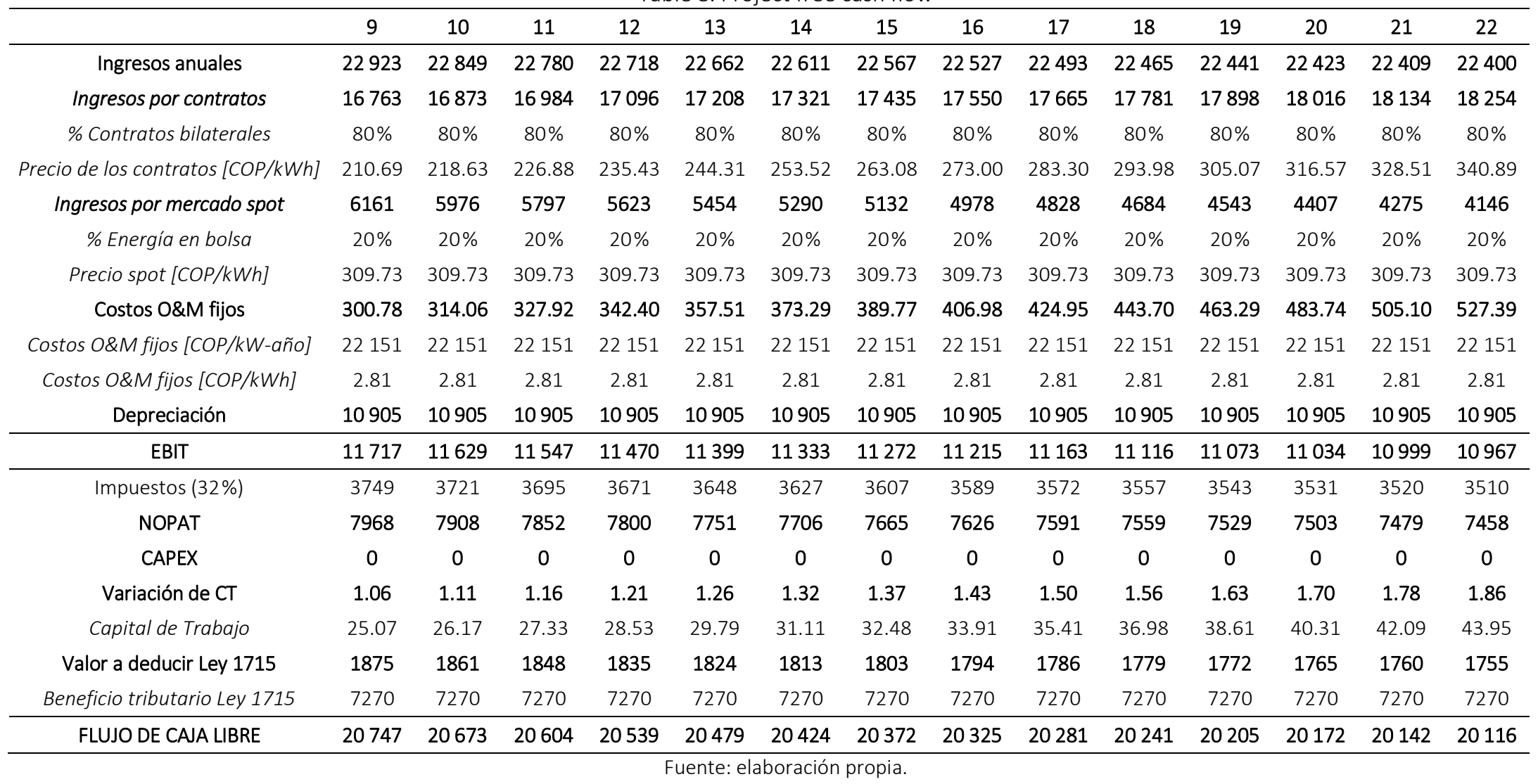

Cifras en MM COP a menos que se indique lo contrario. 